\title{
Implementation of Multiple Output Converters using Time Division Multiple Control with Soft switching and EMI Filter
}

\author{
P.Anisa Begum ${ }^{1}$, E.Thiruvikram², G.Arun sankar ${ }^{3}$ \\ ${ }^{1} P G$ Scholar EEE Department, Gojan School of Business \& Technology, Chennai, Tamil Nadu \\ ${ }^{2}$ Assistant Professor EEE Department, Gojan School of Business \& Technology, Chennai, Tamil Nadu \\ ${ }^{3}$ Associate Professor, EEE Department, Gojan School of Business \& Technology, Chennai, Tamil Nadu
}

\begin{abstract}
Multiple output converters (MOCs) are widely used in equipment for industrial, commercial, and military application like the voltage regulator modules in portable electronic devices. They are preferred due to their advantages in cost, volume, and efficiency. However, most of the MOCs developed so far have limitations in terms of the number of outputs, the tight regulation of all the outputs and the ease of control. In this letter, a novel time division multiple control (TDMC) method which can regulate all of the outputs with high precision is proposed. The proposed charger is able to charge three different kinds of batteries in different state of charges (SOCs) independently and accurately with the constant current/constant voltage (CC/CV) charge method. Also it can offer a high efficiency due to the available soft switching condition at all the switches and EMI filter for reduction of unwanted harmonics.
\end{abstract}

Keywords: TDMC, Multiple output converter, Half bridge inverter, Boost Converter, Voltage doubler and ZVS-ZCS

\section{Introduction}

MOST electrical equipment requires many different voltage levels to take care of their operation so as to generate various types of multiple output converters (MOCs) with several secondary circuits based on every secondary windings are generally considered due to their benefits in terms of cost, volume, and potency. However most of the MOCs developed to date will hardly regulate all of the outputs with a good degree of tight regulation while not the value of quality within the structure and control.Some studies on this subject are conducted and several other strategies are projected like the cross regulation technique [1]-[3], the post regulation technique [4]-[5], and also the PWM/PFM hybrid technique [6]. The cross regulation technique, that regulates solely the most output voltage, is widely used as a result of the simplicity of its structure and control, however the regulation for the rest of the output voltages is not good. In the post regulation technique, an additional switch is employed at every output so as to control all of the output exactly [4]-[6]. However, this technique needs as several controllers because the range of the outputs, that causes complexness within the structure and control. The PWM/PFM hybrid technique will control two outputs independently supported the multiple-band modulation and demodulation, operated by superposed sinusoidal pulse width modulation, pulse frequency modulation, and band-pass filtering [5]. However, this technique is extremely advanced in terms of control, and also the filter related to the rectifier at every output will increase the cost and reduce the efficiency.

Furthermore, all of the above methods require as many secondary windings in the high-frequency transformer as the number of outputs when applied to isolated converter topologies. In this letter, a novel time division multiple control (TDMC) method which can precisely and independently control multiple outputs with only one secondary winding with half bridge topology and obtain boost and buck outputs with high efficiency.

\section{Time Division Multiple Control (TDMC)}

Time-division multiple control (TDMC) is a method of putting multiple data streams in a single signal by separating the signal into many segments, each having a very short duration. Fig. 1 shows the novel TDMC method for MOCs, in which an additional active switch is placed in each secondary output circuit in order to regulate all of the outputs precisely and independently with PWM waveforms for the primary and secondary switches.. As a result, a multiple output battery charger which requires strict voltage and current regulation at the output can be one of the most promising applications for the TDMC method due to its advantages in terms of precise and independent regulation of each output. PWM is performed at the frequency of $f s$ for the primary switch and at $\boldsymbol{f} \boldsymbol{s} / \boldsymbol{n}$ for each of the secondary switches. It regulates all the outputs in one sampling time ( $\boldsymbol{n} \mathbf{T s})$. Each output is controlled during one switching period (Ts). One secondary switch is turned on to allow the primary switch to control each of the outputs. 


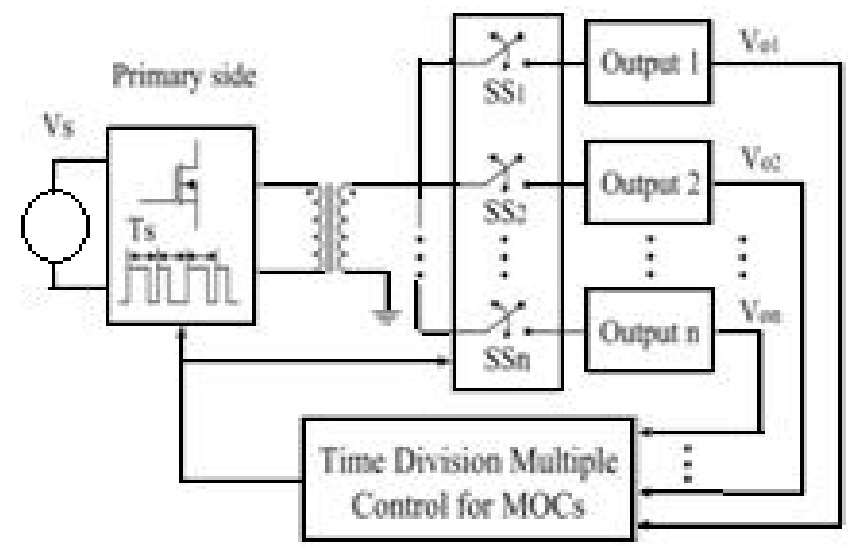

Fig.1.TDMC Method

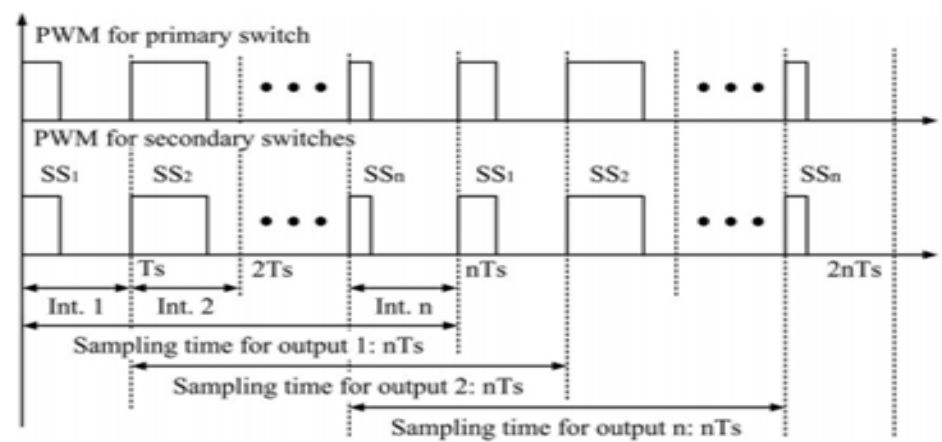

Fig.2. PWM Waveforms of TDMC Method

\section{Proposed System With TDMC}

Time Division Multiple Control (TDMC) method, which can regulate all of the outputs with a high accuracy, is used for a multiple output battery charger based on the half bridge topology. Following are the advantages

- Accurate and independent regulation of each output

- Variable outputs (Buck / Boost)

- No cross regulation problem among outputs

- Only one secondary winding.

- Soft switching by ZVS / ZCS technique

- High Power factor correction

- High and low power applications

- Less components \&Reduced complexity.

- $\quad$ Reduced Transformer size

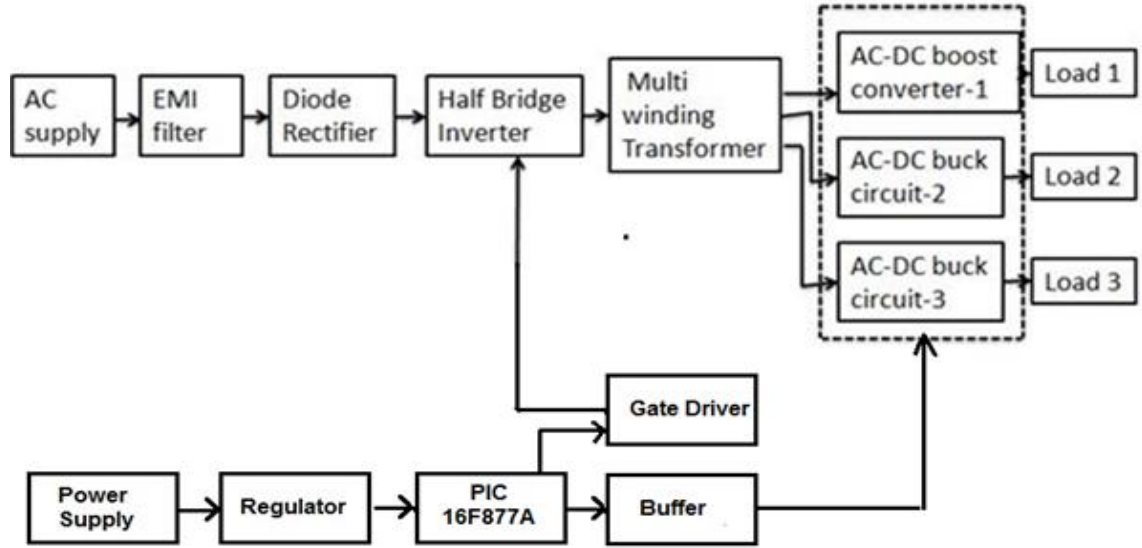

Fig.3. Block diagram of proposed method 
Primary circuit consists of an EMI filter, diode rectifier \& Half bridge inverter circuit. EMI filter used in the modified circuit, is a LC type filter that corrects the power factor so as to keep the voltage \& current in phase and ensure to provide high efficiency. Its function is to reduce high frequency electronic noise that may cause interference with other devices. Regulatory standards exist in most countries that limit the amount of noise that can emitted. EMI, or Electro-Magnetic Interference, is defined as unwanted electrical signals and can be in the form of conducted or radiated emissions. Conducted EMI is where the noise travels along the electrical conductors and radiated EMI is where the noise travels through the air as magnetic fields or radio waves. They are useful in increasing the efficiency of the output

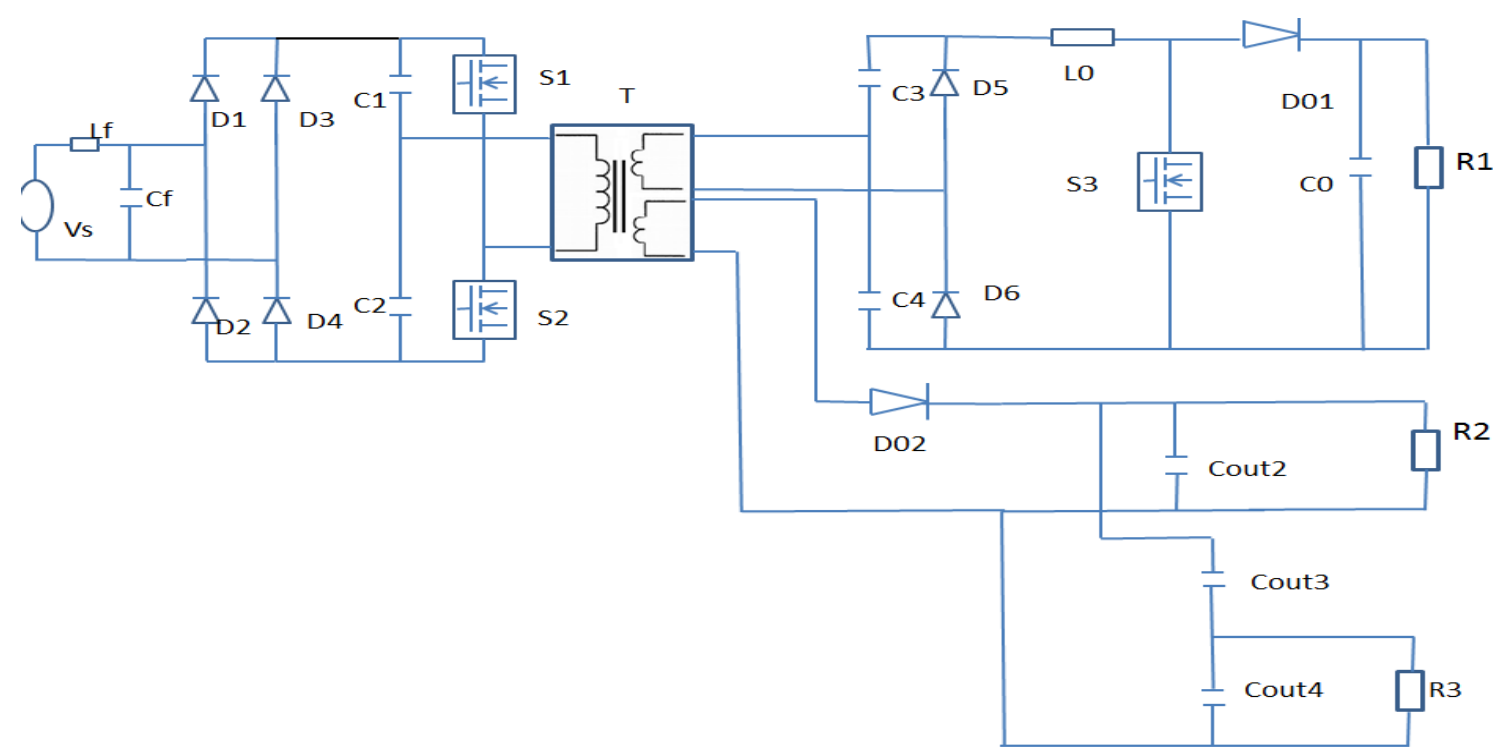

Fig.4. Circuit diagram of proposed method

An EMI filter for a power supply normally consists of passive components, including capacitors and inductors, connected together to form LC circuits. The inductor(s) allow DC or low frequency currents to pass through, while blocking the harmful unwanted high frequency currents. The capacitors provide a low impedance path to divert the high frequency noise away from the input of the filter, either back into the power supply, or into the ground connection.

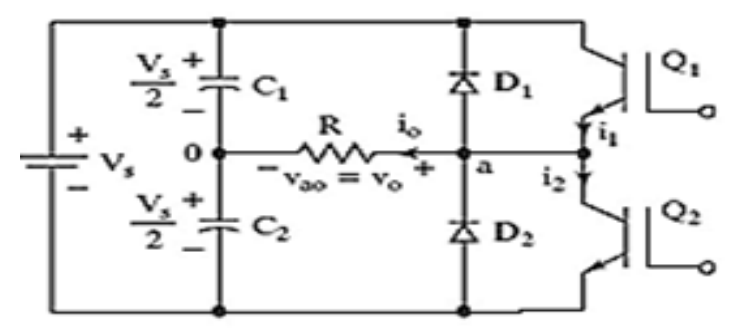

Fig.5. Half bridge inverter

For the Half-bridge inverter circuit as in fig 5, the center-tap of the DC supply is used as one of the load terminals. The center-tap is created by the two series-connected equal-valued capacitors across the DC supply. The dc rail voltages are thus at $+V_{s} / 2$ and $-V_{s} / 2$ with respect to some fictitious ground potential. The two switches, Q1 and Q2, are switched alternately, in a complementary fashion, at the desired output frequency, $f_{\mathrm{o}}$. Here the circuit involves MOSFET as S1 \& S2. An isolation transformer is a transformer used to transfer electrical power from a source of alternating current (AC) power to some equipment or device while isolating the powered device from the power source, usually for safety reasons without electrically connecting the two circuits. The Secondary side circuit consists of 3 different circuits that are supplied by transformer secondary for providing voltages at Load1, Load2 and Load3 respectively For Load 1 output voltage, there is a Voltage doubler \& Boost converter circuits will be used. For Load 2 output voltage, a half wave rectifier circuit will be used. For Load 3 output voltage, an capacitive voltage divider circuit will be used. 
As the name suggests, it produces voltage which is almost twice than the input voltage. This means that the voltage generated by the doubler is twice the peak value of the alternative input current. However note that this is possible only if the load current is very small.Voltage doubler is basically a circuit, consisting of two semi- conductor PN diodes (rectifiers) and two capacitors, arranged in the form of bridge construction.

The Voltage doubler circuit is used to produce a higher voltage direct current from a lower voltage alternating current. It consists of two capacitors \& two diodes. The two capacitors, C1 and C2 are connected in series with each other and also with the load. In voltage doubler circuit the alternating current will first flow in a clockwise pattern and then in anti clockwise (Indicated by arrows in the diagram).

When the current flows in a clockwise direction, the rectifier R1 will charge the capacitor $\mathrm{C} 1$ till the voltage reaches the peak of the positive voltage wave. In the similar manner, then the current flows in an anticlockwise direction, the rectifier R2 will charge the capacitor $\mathrm{C} 2$, till the voltage reaches the peak of the negative voltage wave.

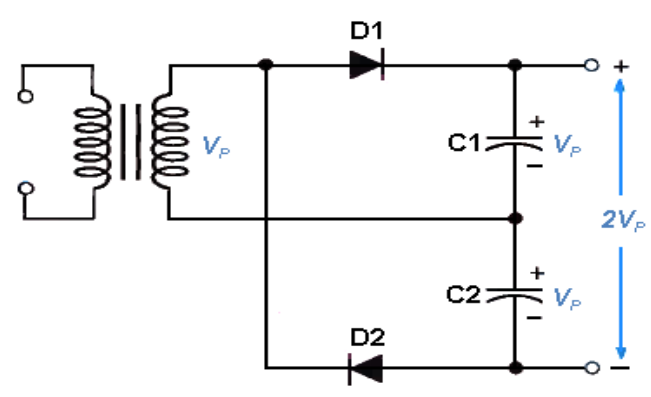

Fig.6. Voltage doubler

A Boost converter is a switch mode DC to DC converter in which the output voltage is greater than the input voltage. It is also called as step up converter.

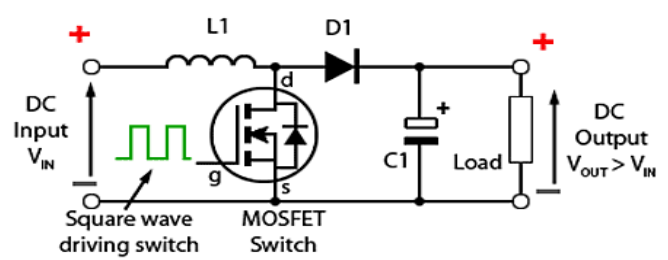

Fig.7. Boost Converter

The main working principle of boost converter is that the inductor in the input circuit resists sudden variations in input current. When switch is OFF the inductor stores energy in the form of magnetic energy and discharges it when switch is closed. The capacitor in the output circuit is assumed large enough that the time constant of RC circuit in the output stage is high. The large time constant compared to switching period ensures a constant output voltage $\mathrm{V}_{\mathrm{o}}(\mathrm{t})=\mathrm{V}_{\mathrm{o}}$ (constant)

The Half wave rectifier is a circuit, which converts an ac voltage to dc voltage. The AC supply to be rectified is generally given through a transformer. The transformer is used to step down or step up the main supply voltage as per the requirement. It also isolates the rectifier from power lines and thus reduces the risk of electric shock. The Pulse Width Modulation is a technique in which the ON time or OFF time of a pulse is varied according to the amplitude of the modulating signal, keeping the (ON time + OFF time) time of the pulse as constant. The (ON time + OFF time) of a pulse is called 'Period' of the pulse, and the ratio of the ON time or OFF time with the Period is called the 'Duty Cycle'. Pulse generation circuit generates the pulses.

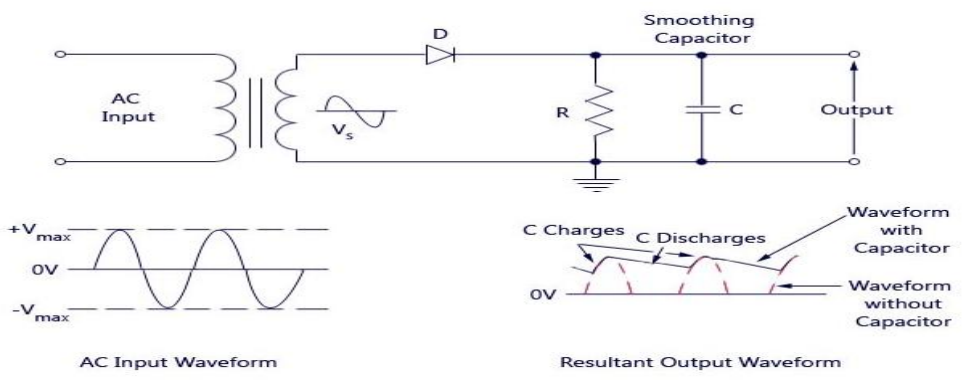

Fig 8 Half wave rectifier 
A voltage divider (also known as a potential divider) is a passive linear circuit that produces an output voltage (Vout) that is a fraction of its input voltage (Vin). Voltage division is the result of distributing the input voltage among the components of the divider.

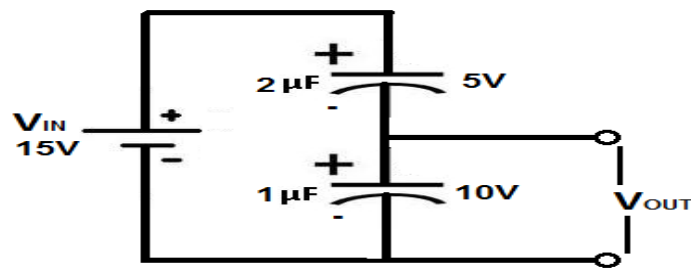

Fig.9. Voltage divider circuit

A capacitive voltage divider is a voltage divider circuit using capacitors as the voltage-dividing components. The common type of voltage divider circuit is one which uses resistors to allocate voltage to different parts of a circuit. This circuit is used to obtain the output voltage at Load 3. Soft switching is a possible way of reducing losses in power electronic switches. The expression "soft switching" actually refers to the operation of power electronic switches as zero-voltage switches (ZVS) or zero-current switches (ZCS).Switching transitions occur under favorable conditions - device voltage or current is zero Reduced switching losses, switch stress, possibly low EMI, easier thermal management. Must for very high frequency operation, (also medium frequency at high power levels).ZVS Switching includes Switch voltage brought to zero before gate voltage is applied, Ideal, zero-loss transition, Low-loss transition, Parallel capacitor as a lossless snubber and Preferred scheme for very high frequency applications using MOSFETs. ZCS Switching at Turn off condition, the Switch current will be brought to zero before gate voltage is removed and it is Ideal, zero-loss transition.

\section{Simulation Analysis}

With the help of MATLAB Tools, the simulation circuit is been built and scopes are implemented to monitor the electrical parameters. The MATLAB platform is optimized for solving engineering and scientific problems. The Simulation result shows the improvement in the waveforms with the modified system. AC $24 \mathrm{~V}$ is given at the primary side and it is been rectified by a diode rectifier to $20 \mathrm{~V} \mathrm{DC}$. Then it is been converted to $20 \mathrm{~V}$ AC using Half wave inverter. Isolation transformer isolates the secondary circuits from the Alternating source. The secondary of the transformer supplies for 3 different circuits to provide Load 1, Load 2 and Load 3 voltages respectively. Fig 10 shows the input voltage \& current of modified circuit. The input voltage is alternating and is in phase with current after passed through EMI filter.

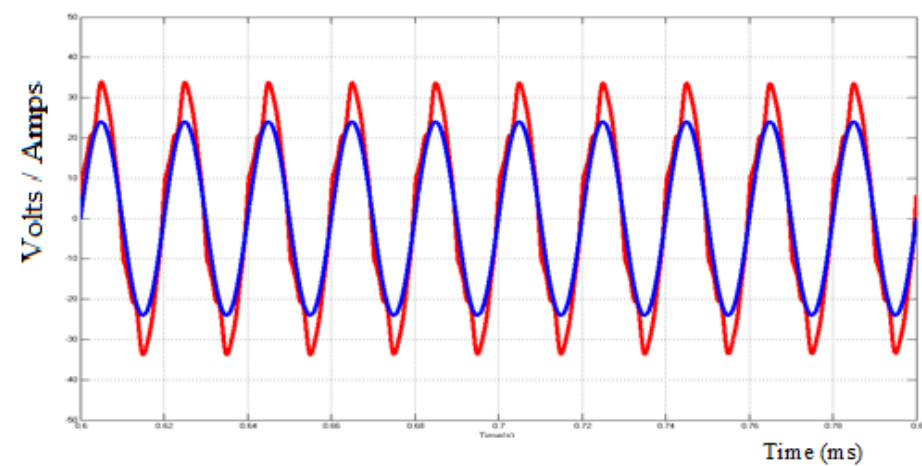

Fig.10. Input voltage\& current of Proposed circuit

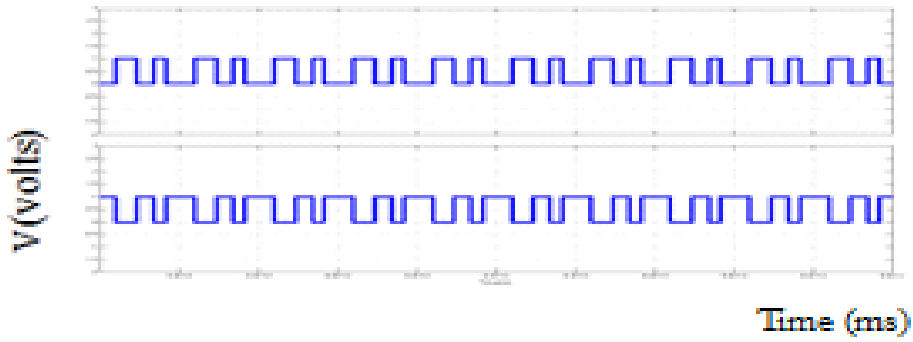

Fig.11. Gate pulses 


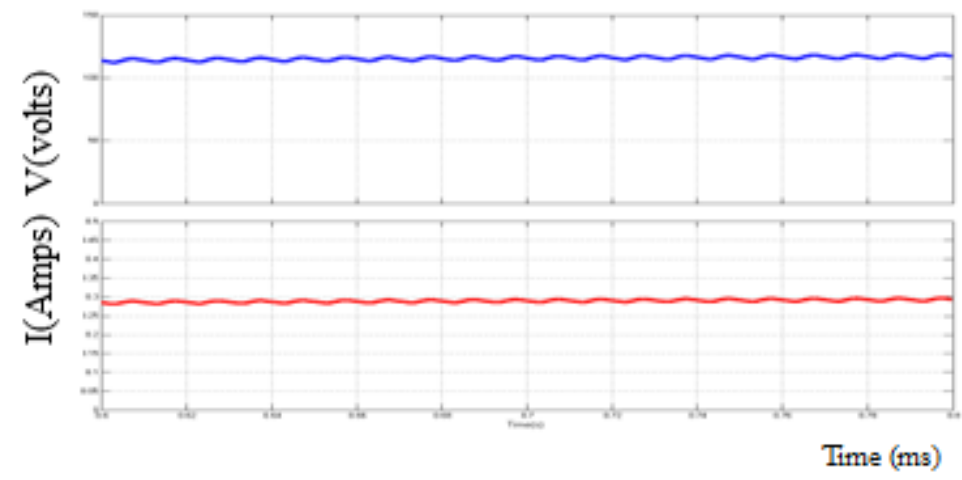

Fig.12. Output Voltage 1

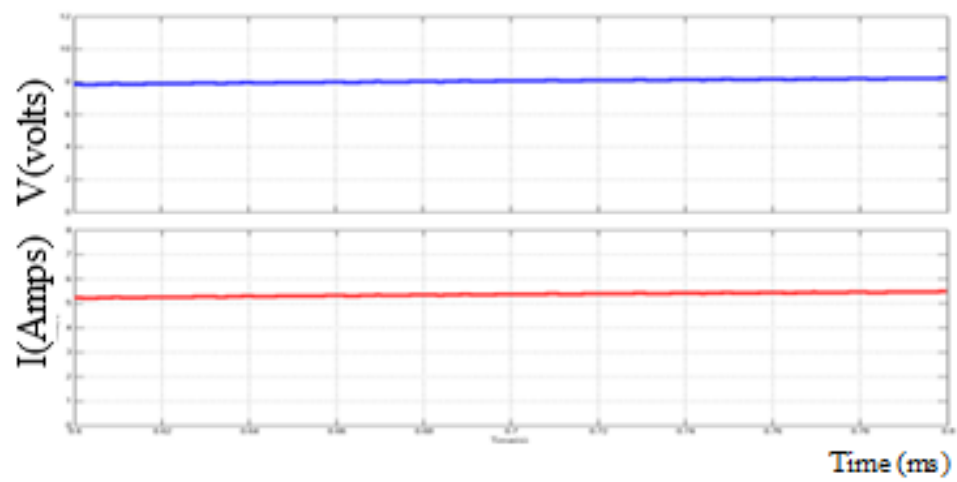

Fig.13. Output Voltage 2

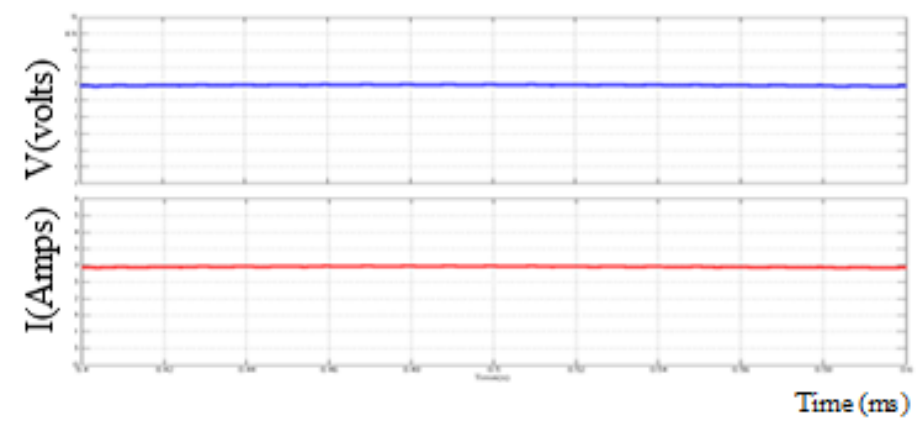

Fig.14. Output Voltage 3

The Hardware circuit is designed to switch the MOSFETs at the desired frequency by programming the PIC controller. Pulse generation circuit has been designed with Power supply, PIC, Buffer amplifier and Gate driver etc. Microcontroller PIC16F877A is one of the PICMicro Family microcontroller. It uses FLASH memory technology so that it can be write-erase until thousand times. A voltage buffer amplifier is used to transfer a voltage from a first circuit, having a high output impedence level, to a second circuit with a low input impedence level. The 74ALS244A is an octal buffer that is ideal for driving bus lines or buffer memory address registers. The outputs are all capable of sinking $24 \mathrm{~mA}$ and sourcing up to $15 \mathrm{~mA}$, producing very good capacitive drive characteristics. The device features two output enables, $\mathrm{OEa}$ and $\mathrm{OEb}$, each controlling four of the 3-State outputs.IRF840 is N-Channel enhancement mode silicon gate power field effect transistor is an advanced power MOSFET designed, tested, and guaranteed to withstand a specified level of energy in the breakdown avalanche mode of operation. All of these power MOSFETs are designed for applications such as switching regulators, switching converters, motor drivers, relay drivers, and drivers for high power bipolar switching transistors requiring high speed and low gate drive power. A gate driver is a power amplifier that accepts a low-power input from a controller IC and produces a high-current drive input for the gate of a highpower transistor such as an IGBTor power MOSFET and it helps to switch the primary side MOSFETs S1 and S2. The IR2110/IR2113 are high voltage, high speed power MOSFET and IGBT drivers with independent high and low side referenced output channels. Secondary side MOSFET S3 is switched through the optocoupler. 


\section{Conclusion}

The proposed method offers a simple control technique to achieve a tight regulation for all of the outputs and can be applied to all kinds of isolated converter topologies, with a high accuracy, and is used for a multiple output battery charger Number of components are reduced and thereby overall cost of the system. The overriding purpose of this study is to achieve high efficiency multiple outputs using Time Division Multiple Control (TDMC) method, which can regulate all of the outputs with a high accuracy and thereby obtain three different outputs that includes one high gain output \& two low gain output.

\section{References}

[1]. Reddy. J, Bhuvaneswari. G, and Singh. B, “A Single DC-DC Converter Based Multiple Output SMPS with Fully Regulated and Isolated Outputs", INDICON, 2005 Annual IEEE, December 2005, pp: 585 589

[2]. J. Chuanwen, M. Smith, Jr., K. M. Smedley, and K. King, "Cross regulation in flyback converters: analytic model and solution," Power Electronics, IEEE Transactions on, vol. 16, pp. 231-239, 2001.

[3]. P. Patra, J. Ghosh, and A. Patra, "Control Scheme for Reduced Cross-Regulation in Single-Inductor Multiple-Output DC-DC Converters," Industrial Electronics, IEEE Transactions on, vol. 60, pp. 5095-5104, 2013.

[4]. B. Su, H. Wen, J. Zhang, and Z. Lu, "A soft-switching post-regulator for multi-outputs dual forward DC/DC converter with tight output voltage regulation," Power Electronics, IET, vol. 6, pp. 1069-1077, 2013.

[5]. S. Zhonghan, C. Xuegui, W. Weiwei, T. Xi, Y. Na, and M. Hao," Predictive Digital Current Control of Single-Inductor MultipleOutput Converters in CCM With Low Cross Regulation”, Power Electronics, IEEE Transactions on, vol. 27, pp. 1917-1925, 2012

[6]. Van-Long Tran, and Woojin Choi, "Novel Time Division Multiple Control method for Multiple Output Battery Charger", Power Electronics, IEEE Transactions on, vol. 29, issue. 10, pp. $5102-5105$

[7]. W. Hwa-Young, C. Soo-Yong, S. Young-Min, C. Dae- Taek, and H. Soon-Chan, "Analysis of the lithium battery charge/discharge system using state space averaging method," in Power Electronics and Motion Control Conference, 2009. IPEMC '09. IEEE 6th International, 2009, pp. 1402-1406.

[8]. J. Van Mierlo, P. Van den Bossche, and G. Maggetto, "Models of energy sources for EV and HEV: fuel cells, batteries, ultracapacitors, flywheels and engine-generators," Journal of Power Sources, vol. 128, pp. 76- 89, 3/29/ 2004.

[9]. N. B. Hadj-Youssef, K. Al-Haddad, H. Y. Kanaan, and F. Fnaiech, "Small-signal perturbation technique used for DSP-based identification of a three-phase three-level boost-type Vienna rectifier," Electric Power Applications, IET, vol. 1, pp. 199-208, 2007.

[10]. G. Sen and M. E. Elbuluk, "Voltage and Current- Programmed Modes in Control of the Z-Source Converter," Industry Applications, IEEE Transactions on, vol. 46, pp. 680-686, 2010 